\title{
TITLE:
}

\section{Characterization of ablated species in laser-induced plasma plume}

$\operatorname{AUTHOR}(S)$ :

Furusawa, H; Sakka, T; Ogata, YH

CITATION:

Furusawa, H ...[et al]. Characterization of ablated species in laserinduced plasma plume. JOURNAL OF APPLIED PHYSICS 2004, 96(2): 975-982

ISSUE DATE:

2004-07-15

URL:

http://hdl.handle.net/2433/50403

\section{RIGHT:}

Copyright 2004 American Institute of Physics. This article may be downloaded for personal use only. Any other use requires prior permission of the author and the American Institute of Physics. 


\title{
Characterization of ablated species in laser-induced plasma plume
}

\author{
Hideki Furusawa, Tetsuo Sakka, and Yukio H. Ogata \\ Institute of Advanced Energy, Koto University, Uji, Kyoto 611-0011, Japan
}

(Received 4 December 2003; accepted 14 April 2004)

\begin{abstract}
Plasma electron density and atomic population densities in the plasma plume produced by a laser ablation of aluminum metal were determined in various ambient gases at relatively high pressures. The method is based on the fit of a spectral line profile of $\mathrm{Al}(\mathrm{I}){ }^{2} P^{\circ}-{ }^{2} S$ emission to the theoretical spectrum obtained by one-dimensional radiative transfer calculation. The electron density was higher for a higher ambient gas pressure, suggesting the confinement of the plume by an ambient gas. The electron density also depends on the type of ambient gases, i.e., it increased in the order $\mathrm{He}<\mathrm{CH}_{4}<\mathrm{N}_{2}<\mathrm{CF}_{4}$, while the atomic population density is almost independent of the type of ambient species and pressure. The population densities of the upper and lower levels of the transition were compared, and the ratio between their spatial distribution widths was calculated. These results provide valuable information regarding the confinement of the plume by the ambient gas and give insight into the time evolution of the plume. (C) 2004 American Institute of Physics. [DOI: $10.1063 / 1.1759397]$
\end{abstract}

\section{INTRODUCTION}

In the process of pulsed laser ablation of solid target, highly energetic species are ejected from the surface. The energy often reaches up to several hundreds of $\mathrm{eV}^{1-3}$ The ejected species form a plasma plume. In the plume, the ablated species undergo various chemical reactions by themselves and also with ambient species. By using these species, thin films ${ }^{4-6}$ or fine particles ${ }^{7-10}$ can be obtained. The combination of various targets and ambient species enables a wide variety of reactions, and hence, the production of various materials. The understanding and the control of the reaction are important to improve the quality of the resultant materials. For these purposes, the investigation for the spatial distribution of the free electron density as well as the excited-atom density is useful.

The ablation has usually been studied under low pressure conditions: up to 1 Torr in pulsed laser deposition of thin films and higher pressures for fine particle formations. We have performed the ablation experiments at much higher pressures up to 1520 Torr, and also in liquids. ${ }^{11-14}$ These conditions have an advantage of confining highly active ablated species into a small region. The confinement effect enhances the collision frequency, and therefore, provides a specific chemical reaction field in the plume. For example, production of novel materials such as carbon nanotubes ${ }^{15,16}$ and diamondlike carbon ${ }^{17,18}$ has been reported. In addition, the confinement effect has a possibility of surface modification by localized reactions on a target surface or selective deposition of the reaction products onto the laser irradiation spot.

The plasma plume is dynamically changing, and therefore, comprehension of the temporal behavior is necessary for understanding the kinetics of ablated species in the

\footnotetext{
a) Author to whom correspondence should be addressed; FAX: +81-774-383499; electronic mail: t-sakka@iae.kyoto-u.ac.jp
}

plume. Atomic populations of the ground and excitation levels, and also the plasma electrons critically affect reaction rates. Electrons ejected from the target have high kinetic energy and ionize or activate ambient species through collisions. The species excited in such processes also participate in various reactions. Therefore, electron density and its spatial distribution as well as those of the atomic and molecular populations are important parameters.

Many studies on the electron density in the plasma plume have been performed, using techniques such as optical emission spectroscopy, ${ }^{19,20}$ the Langmuir probe, ${ }^{21,22}$ interferometry, ${ }^{23,24}$ and optical transmissivity. ${ }^{25}$ The emission spectroscopy has been widely used because of its noncontact nature of probing and the information attainable over a wide spectral range. The electron density has been estimated from the Stark broadening of emission lines in the case of the laser ablation under low pressure conditions. ${ }^{19,20}$

Under high pressure conditions, however, the emission lines with a self-reversed structure are often observed, where the electron density cannot be evaluated simply from the width of the spectral line. The appearance of the selfreversed structure is due not only to a high optical thickness of the plume but also to different spatial distributions for the atomic population densities of the two levels involved in the transition. If the population of the lower level has a larger spatial distribution than that of the upper level, then the selfabsorption in the "cold" peripheral region of the plume will be more pronounced than in the center. Therefore, the position dependence of the self-absorption in the plume as well as the position dependence of the Stark effects has to be considered in order to obtain theoretical spectral line profiles with the self-reversed structure.

In our previous paper, ${ }^{26}$ we proposed a method to evaluate the electron density as well as the atomic population densities of the two levels involved in a transition. In the method they are evaluated as best-fit parameters of the fitting process to a theoretical spectrum. The theory is based on the calcu- 
lation of a spectral line profile by taking account of the spatial distributions of the atomic population of each level independently. The spectral line profile with the self-reversed structure was reproduced successfully by the calculation based on one-dimensional radiative transfer, giving information about the ablated species in the plume: the electron density, the atomic population density, and their spatial distribution width. The self-reversed structure originates from the absorption by the species in the lower level. Therefore, the information obtained by the analysis of the spectral line profile with the self-reversed structure contains the information usually obtained by the emission and absorption measurements.

In this paper, the method is applied to laser ablation plasma plumes produced by using the aluminum metal target in various ambient gases at relatively high pressures. The purpose of the present work is to characterize ablated species in the plume and to clarify the confinement effects of the plume by the ambient gases. Time-resolved analysis is an important aspect of this work. Time evolution of the electrons and ablated species in the plume is shown. Also, reliability of the results obtained by fitting to the theory is discussed.

\section{EXPERIMENT}

The experimental setup consists of a vacuum chamber equipped with a rotary pump for evacuation and a line for gas introduction. $\mathrm{N}_{2}, \mathrm{He}, \mathrm{CF}_{4}$, and $\mathrm{CH}_{4}$ were used as an ambient gas, and the chamber was filled with each of them after evacuation. The pressure was regulated at 760 Torr or 1520 Torr by a capacitance-type pressure gauge. Laser ablation was performed using a Nd:YAG (where YAG stands for yttrium aluminum garnet) laser with the pulse duration of 20 ns, pulse energy of $30 \mathrm{~mJ}$, and wavelength of $1064 \mathrm{~nm}$. The laser beam was focused by a lens with a focal length $f$ $=10 \mathrm{~cm}$, and irradiated onto an aluminum metal $(99.9999 \%$, Nilaco Co.) placed in the chamber.

The emission from the laser-induced plasma plume was imaged onto the entrance slit of the spectrograph. The spectral range was set from $393 \mathrm{~nm}$ to $398 \mathrm{~nm}$ in order to measure $\mathrm{Al}(\mathrm{I})$ transitions, which appear at $396.152 \mathrm{~nm}$ and $394.401 \mathrm{~nm} ; 1 \mathrm{~m}$ focal length double dispersion spectrograph (Ritsu Oyo Kogaku, MC100N) equipped with two 1800 grooves $/ \mathrm{mm}$ diffraction gratings was used. The slit with 20 $\mu \mathrm{m}$ in width was arranged to be parallel to the target surface. An intensified charge coupled device (ICCD) (Princeton Instrument, ICCD-1024MTDGE/1) was used as a detector. The spectral resolution of this system was $0.04 \mathrm{~nm}$. The duration of the time gate to drive the ICCD detector was $20 \mathrm{~ns}$ for optical emission spectroscopy, and the time delay from the laser pulse was varied from 100 to $5000 \mathrm{~ns}$.

The information of the plume size is necessary for the model calculation. Therefore, lateral imaging of the optical emission from the plume was performed using the ICCD camera equipped with a lens (Nikon, AF Nikkor $20 \mathrm{~mm}$ F2.8D) and a bellows attachment (Nikon, PB-6) for magnification. The duration of the gate was set to $5 \mathrm{~ns}$. The width of the plume was obtained by fitting the intensity profile of the image to the Gaussian function.

\section{ANALYSIS OF SPECTRAL LINE PROFILES}

The analysis is based on fitting the experimental line profile to one-dimensional radiative transport calculation, which has been developed in the previous paper. ${ }^{26}$ The appearance of the self-reversed structure suggests that the populations of the levels involved in a transition have different spatial distributions. In the following we briefly explain the model, which has been explained in detail in our previous paper. ${ }^{26}$

For the analysis of the spectral line profile, it must be taken into account that a spectral intensity of the optical emission is a result of the integration along the light propagation line of the observation. The spectral radiant flux density $I(x, \nu)$, as a function of frequency $v$ and the position $x$ along the propagation line, is expressed by the following one-dimensional radiative transfer equation,

$$
d I(x, \nu)=[\epsilon(x, \nu)-\kappa(x, \nu) I(x, \nu)] d x,
$$

where $\epsilon(x, \nu)$ and $\kappa(x, \nu)$ are the spectral emission and absorption coefficients, respectively. In order to calculate $I(\nu)$, functions $\epsilon(x, \nu)$ and $\kappa(x, \nu)$ are necessary. In terms of the Einstein coefficients, $A, B_{21}$, and $B_{12}$, which represent spontaneous emission, induced emission, and absorption, respectively, they are expressed as follows by using a normalized spectral line profile $f(x, \nu)$, i.e.,

$$
\begin{aligned}
& \epsilon(x, \nu)=\frac{A n_{2}(x) h \nu f(x, \nu)}{4 \pi}, \\
& \kappa(x, \nu)=\frac{\left[B_{12} n_{1}(x)-B_{21} n_{2}(x)\right] h \nu f(x, \nu)}{c},
\end{aligned}
$$

where $n_{1}(x)$ and $n_{2}(x)$ are the population densities of the lower and upper levels, respectively, $h$ is the Planck constant, and $c$ is the light velocity. In a plasma plume produced by laser ablation, the Stark effect has to be considered in the determination of $f(x, \nu)$. The effect is usually reduced to a line shift $\Delta \lambda_{\text {shift }}$ and a broadening $\Delta \lambda_{\text {width }}$, which are approximated to be proportional to the electron density $n_{e}(x)$, i.e.,

$$
\begin{aligned}
& \Delta \lambda_{\text {shift }}(x)=d n_{e}(x), \\
& \Delta \lambda_{\text {width }}(x)=w n_{e}(x),
\end{aligned}
$$

where $d$ and $w$ are the Stark shift and width parameters, respectively. Note that the electron density $n_{e}(x)$ is a function of $x$, since we consider a distribution of the plasma electrons. By assuming the Lorentzian profile of $f(x, \nu)$, we obtain

$$
f(x, \nu)=\frac{1}{\pi} \frac{\Delta \nu_{\text {width }}(x)}{\left[\Delta \nu_{\text {width }}(x)\right]^{2}+\left[\nu-\nu_{0}+\Delta \nu_{\text {shift }}(x)\right]^{2}},
$$

where $\nu_{0}$ is the unperturbed resonant frequency, $\Delta \nu_{\text {shift }}$ $=\left(c / \lambda_{0}^{2}\right) \Delta \lambda_{\text {shift }}(x)$, and $\Delta \nu_{\text {width }}=\left(c / \lambda_{0}^{2}\right) \Delta \lambda_{\text {width }}(x)$. As for the spatial distribution functions $n_{e}(x), n_{1}(x)$, and $n_{2}(x)$, we assumed the Gaussian distributions, because an intensity profile of the plume image was approximately 
Gaussian. By letting the point at $x=0$ be a center of the plume, they were assumed independently as follows:

$$
\begin{aligned}
& n_{e}(x)=N_{e 0} \exp \left(-\frac{x^{2}}{\sigma_{e}^{2}}\right), \\
& n_{1}(x)=N_{10} \exp \left(-\frac{x^{2}}{\sigma_{1}^{2}}\right), \\
& n_{2}(x)=N_{20} \exp \left(-\frac{x^{2}}{\sigma_{2}^{2}}\right),
\end{aligned}
$$

where $\sigma_{e}, \sigma_{1}$, and $\sigma_{2}$ are the width parameters for the electron density distribution and for the population densities of the lower and upper levels, respectively, and $N_{e 0}, N_{10}$, and $N_{20}$ are their maximum densities at $x=0$, respectively. By substituting Eqs. (6)-(9) into Eqs. (2) and (3) we obtain $\epsilon(x, \nu)$ and $\kappa(x, \nu)$. Then Eq. (1) is integrated numerically to obtain $I(v)$. Finally, the convolution with an instrumental function is performed to obtain a spectrum to be compared with the experimental spectrum.

For the calculation it is necessary to determine the parameters $\sigma_{e}, \sigma_{1}, \sigma_{2}, N_{e 0}, N_{10}$, and $N_{20}$. In this work, the six parameters were reduced to three adjustable parameters, namely $N_{e 0}, N_{10}$, and $\sigma_{1}$, by the following assumptions. The first assumption is $\sigma_{e}=\sigma_{2}$, i.e., both plasma formation and the formation of the upper-level atoms occur in the same region. Furthermore, the value of $\sigma_{2}\left(=\sigma_{e}\right)$ was assumed to be the 1/e width of the Gaussian distribution best fitted to a lateral intensity profile of the emission image of the plume. Finally, the population ratio at the plume center was fixed by giving a certain value to $T_{0}$ in the following equation:

$$
\frac{N_{20}}{N_{10}}=\frac{g_{2}}{g_{1}} \exp \left(-\frac{h \nu_{0}}{k_{B} T_{0}}\right)
$$

where $k_{B}$ is the Boltzmann constant, and $g_{2}$ and $g_{1}$ are the degeneracy of the upper and lower levels, respectively. We examined the effect of the $T_{0}$ values, and found that we always obtain virtually the same best-fit parameters when $2000 \mathrm{~K}<T_{0}<20000 \mathrm{~K}$. This means that $N_{20}$ does not influence the spectral profile as long as the ratio $N_{20} / N_{10}$ is in a certain range. Although the spatial variation of the two-level temperature can be discussed through the resultant $\sigma_{1}$ and $\sigma_{2}$ values, we cannot obtain the temperature quantitatively.

In the present work, the model was applied to the emission lines of ${ }^{2} P^{\circ}{ }_{-}^{2} S$ transitions of $\mathrm{Al}(\mathrm{I})$ in the plume. The lower state or the ground state has a fine structure doublet, ${ }^{2} P_{3 / 2}^{\circ}$ and ${ }^{2} P_{1 / 2}^{\circ}$. Therefore, two transition lines appear at $396.152 \mathrm{~nm}\left({ }^{2} P_{3 / 2}^{\circ}{ }^{2} S_{1 / 2}\right)$ and $394.401 \mathrm{~nm}\left({ }^{2} P_{1 / 2}^{\circ}{ }^{2} S_{1 / 2}\right)$. We introduce suffixes " $a$ " and " $b$ " for ${ }^{2} P_{3 / 2}^{\circ}$ and ${ }^{2} P_{1 / 2}^{\circ}$, respectively. The energy difference between the ground state fine structure levels is very small $\left(112 \mathrm{~cm}^{-1}\right)$, so that we assume the ratio $N_{1 a} / N_{1 b}$ to be the ratio of the degeneracy throughout the spatial region of the calculation, and hence, leading to $\sigma_{1 a}=\sigma_{1 b}$. Therefore, in the case of this system, $N_{1 b}$ and $\sigma_{1}$ can be the representative parameters for the ground states.

The calculation was iteratively performed by a personal computer until the best-fit spectral line profile was obtained. The fitting process was applied to all the spectral line profiles

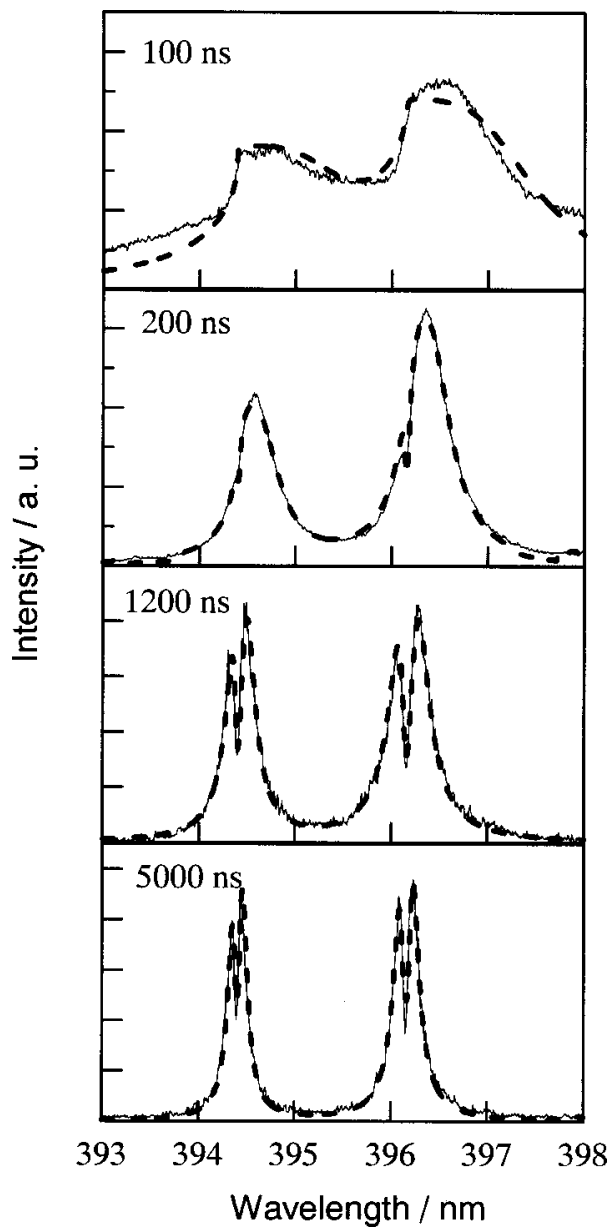

FIG. 1. Spectral line profiles of $\mathrm{Al}(\mathrm{I})$ transitions at each delay time observed at 760 Torr of $\mathrm{N}_{2}$ gas. Solid and broken lines represent observed and calculated profiles, respectively.

at various delay times, which enabled the clarification of the time evolution as well as spatial distribution of the electron density and the population densities of ablated species in the plume.

\section{RESULTS}

In $\mathrm{N}_{2}, \mathrm{CF}_{4}$, and $\mathrm{CH}_{4}$ gases, clear emission lines of $\mathrm{Al}(\mathrm{I})$ transitions were not observed until $200 \mathrm{~ns}$ after the irradiation because of a strong continuous spectral radiation, while in He gas, clear emission lines were observed even at $100 \mathrm{~ns}$ after the irradiation. As long as clear emission lines were observed, the self-reversed structure was seen and the intensities of the doublet lines were nearly equal in every gas atmosphere. The spectral line profiles observed in $\mathrm{N}_{2}$ gas atmosphere are shown in Fig. 1. The profiles were always well reproduced by the model calculation, except for the profiles observed in a very early stage, as shown in the top figure in Fig. 1. The discrepancy in the early stage is because the continuous radiation is not taken into account in the calculation. With increasing time, asymmetry of each line became less pronounced, and the dip position with respect to the whole line profile moved to the line center. In addition, the spectral line broadening became smaller with increasing 


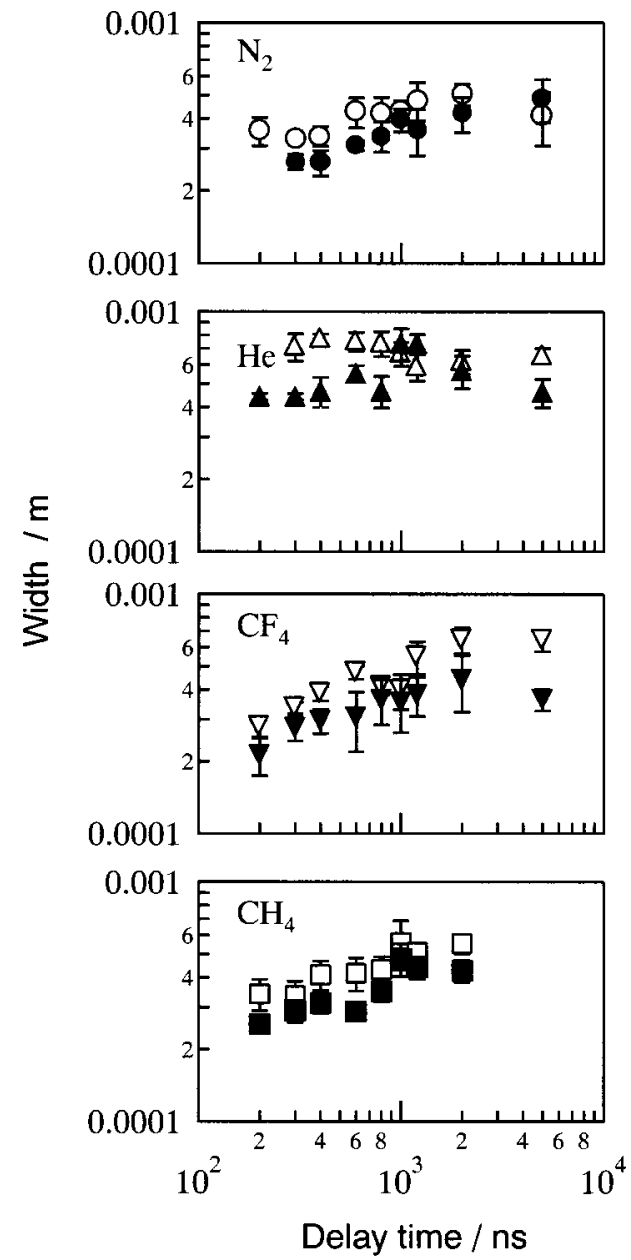

FIG. 2. The $1 / e$ width derived by fitting lateral intensity profiles of the plume to the Gaussian distribution in each gas. Open symbols represent data at 760 Torr, and closed at 1520 Torr. The results were used for the $\sigma_{2}$ parameter in the theoretical calculation.

time. In He gas atmosphere, the spectral line broadening was smaller than in any other gases, and the self-reversed structure was less distinguished.

The time evolution of the plume size is shown in Fig. 2. The results shown in this figure were used as $\sigma_{2}\left(=\sigma_{e}\right)$ in the theory. The intensity profiles of the lateral images of the plume at each delay time fitted well to Gaussian. The lateral width of the plume was obtained as a 1/e width. In every gas atmosphere, higher gas pressure gives smaller width, suggesting that the plume expansion is suppressed. In $\mathrm{N}_{2}, \mathrm{CF}_{4}$, and $\mathrm{CH}_{4}$ gases, the plume size increased gradually until 1000 ns after the irradiation, and then saturated. In the $\mathrm{He}$ gas atmosphere, the time evolution of the plume was different from that in the other gases. At both pressures the plume size was the largest when He was used as an ambient gas.

The results of electron density parameter $N_{e 0}$ obtained through the fitting process are given in Figs. 3 and 4. Figure 3 shows the effect of ambient gases on $N_{e 0}$. The initial values of the order of $10^{24} \mathrm{~m}^{-3}$ are in agreement with those given in previous reports. ${ }^{19-21,25} N_{e 0}$ decreased drastically with increasing time till $2000 \mathrm{~ns}$, while later no significant decrease was observed regardless of the ambient gases and their pressure. Furthermore, $N_{e 0}$ varied with the type of at-

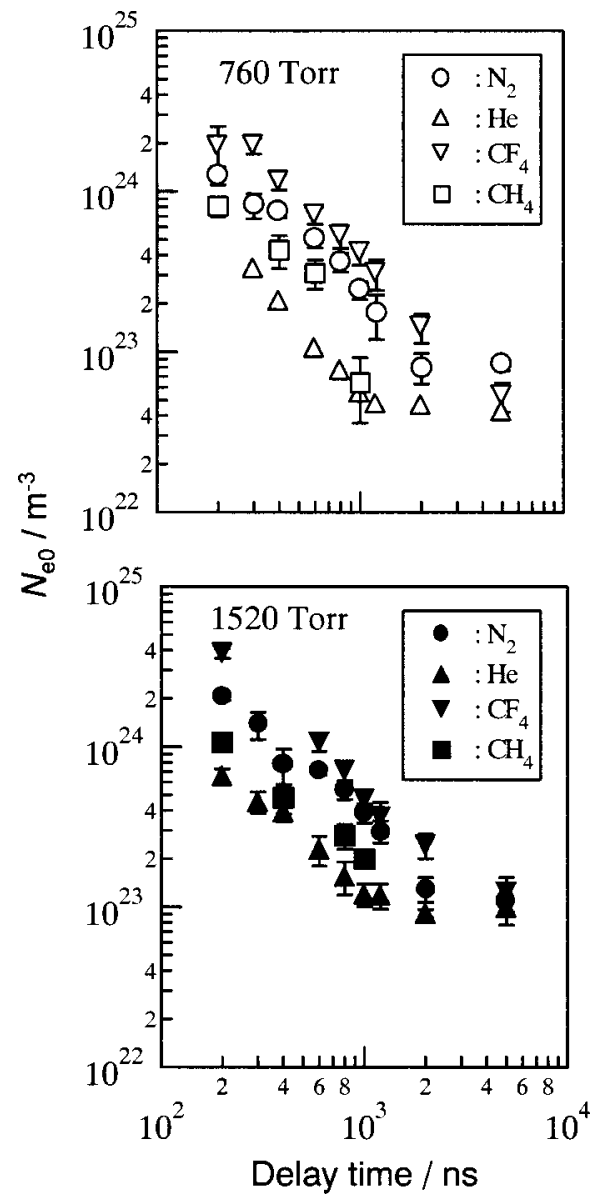

FIG. 3. Electron density parameter $N_{e 0}$ obtained at 760 and 1520 Torr.

mosphere, i.e., $\mathrm{He}<\mathrm{CH}_{4}<\mathrm{N}_{2}<\mathrm{CF}_{4}$ throughout the observation time range. The difference between the electron densities at 760 Torr and 1520 Torr is shown in Fig. 4. Higher ambient gas pressure caused higher $N_{e 0}$ values at any delay time. The ambient pressure effect was larger in the early stage. The effect was remarkable in $\mathrm{CF}_{4}$ and $\mathrm{N}_{2}$ gases. After $1000 \mathrm{~ns}$, the effect was nearly the same in any gas.

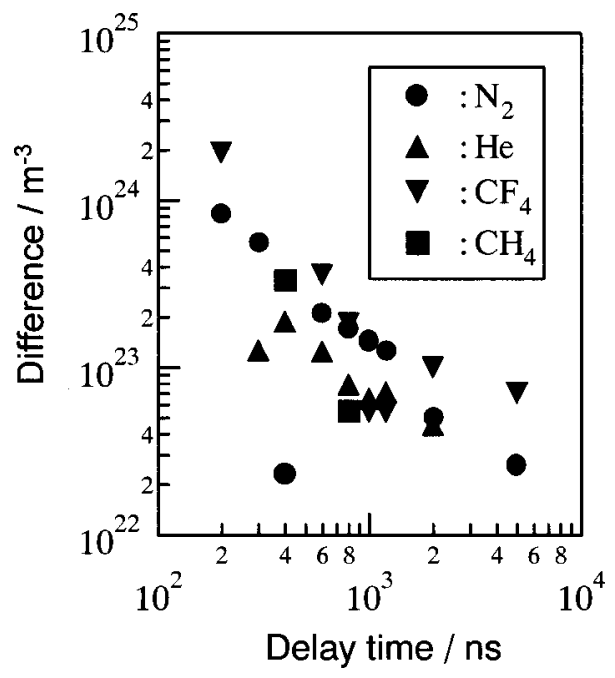

FIG. 4. The difference between $N_{e 0}$ obtained at 760 and 1520 Torr. 


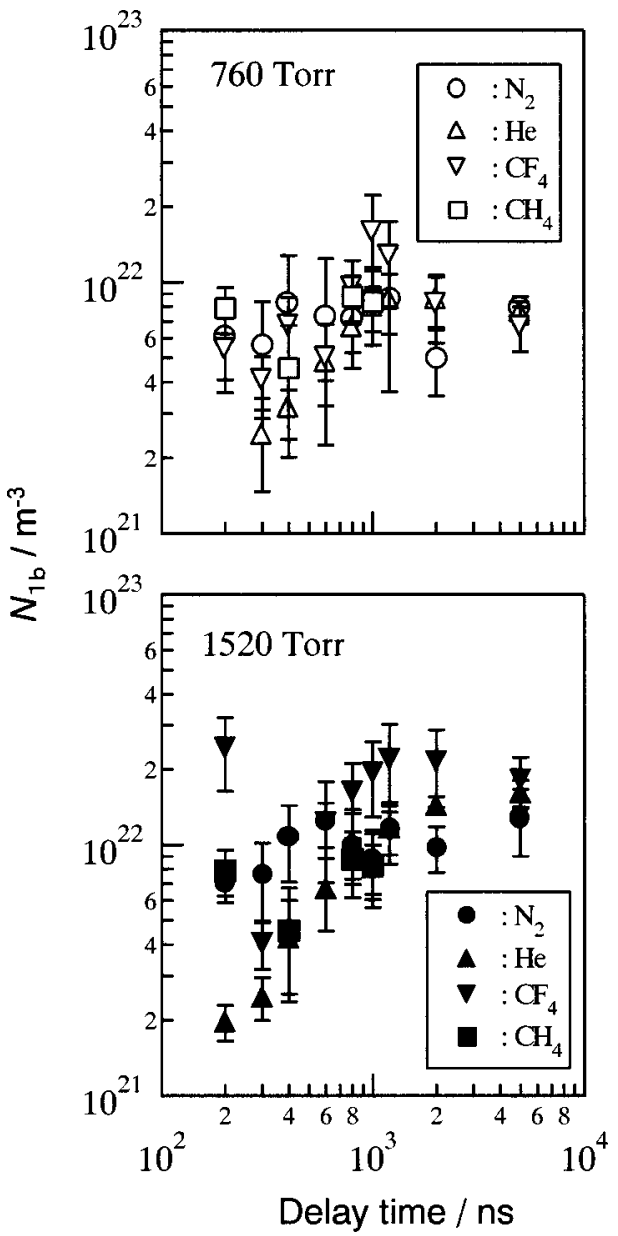

FIG. 5. Atomic population density parameter $N_{1 b}$ obtained at 760 and 1520 Torr.

The results of the other parameters $N_{1 b}$ and $\sigma_{1}$ are shown in Figs. 5 and 6, respectively. The dependence of $N_{1 b}$ and $\sigma_{1}$ upon the type of gases or the pressure was not as clear as $N_{e 0} . N_{1 b}$ increased gradually with time, and then saturated after $1000 \mathrm{~ns}$ to be ca. $1 \times 10^{22} \mathrm{~m}^{-3}$ in 760 Torr and ca. $2 \times 10^{22} \mathrm{~m}^{-3}$ at 1520 Torr. The order of magnitude of these values agreed with an atomic density estimated from the etched amount of the target and the plume volume. The results of $\sigma_{1}$, which represents spatial distribution width of the lower-level population, are shown in Fig. 6. $\sigma_{1}$ seems to increase with time from $0.4 \times 10^{-3} \mathrm{~m}$ up to $1 \times 10^{-3} \mathrm{~m}$ under both pressure conditions. $\sigma_{1}$ at each delay time was larger than $\sigma_{2}$, which suggests that temperature determined by the two levels is higher in the plume center than in the peripheral region.

The results show that the data dispersion of $N_{e 0}$ was very small throughout the observation time range, while that of the other parameters $N_{1 b}$ and $\sigma_{1}$ was comparatively large.

\section{DISCUSSION}

\section{A. Reliability of the best-fit parameters}

In the model, some assumptions have been made in order to decrease the number of adjustable parameters. The

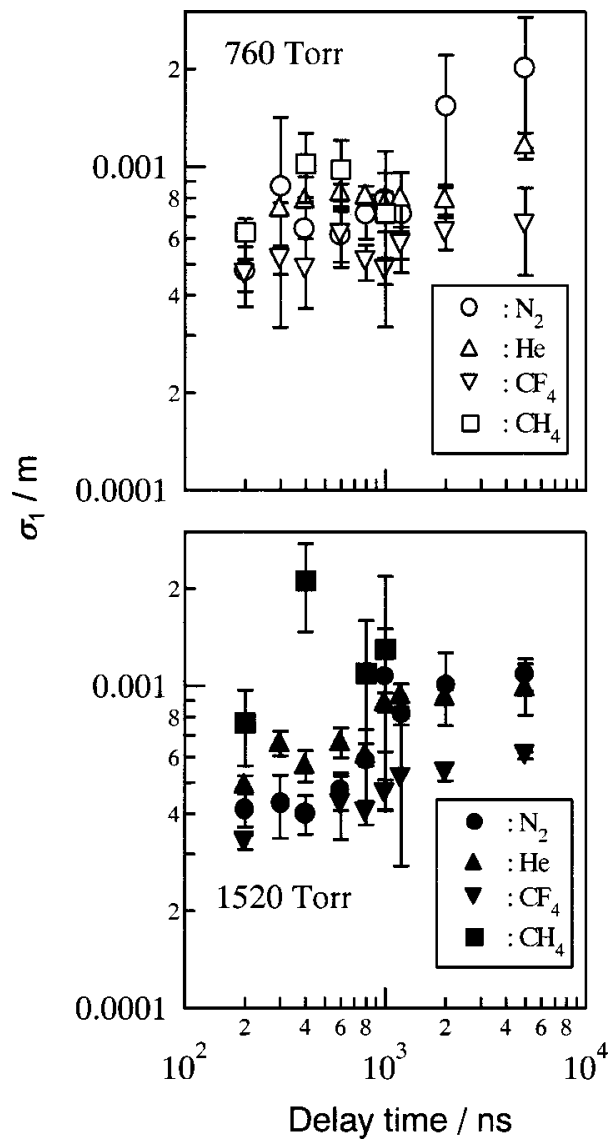

FIG. 6. Spatial distribution parameter $\sigma_{1}$ obtained at 760 and 1520 Torr.

reliability of the best-fit parameters obtained through the calculation owes partly to them. Here, the reliability of the results is discussed.

In the case of laser-induced plasma, where a density of species is very high, the major mechanisms affecting a spectral line profile are the Stark effect and the saturation by self-absorption. The former is due to the high density of plasma electrons and the latter is due to the absorption by the atoms in the lower level of the transition. A higher density of excited atoms brings about a stronger intensity of the spectral line emission. In optically thick plasma, the intensity of the emission line saturates at the luminance of black body, which gives a plateau in the line center. Further stronger emission with self-absorption leads to the saturation in the neighborhood of the line center, and hence, results in a spectral line broadening. On the other hand, the present results show that the ratio of the peak intensity of the two emission lines (394.401 $\mathrm{nm}$ and $396.152 \mathrm{~nm}$ ) was almost unity especially in the time range after a few $100 \mathrm{~ns}$, although the ratio is expected to be 0.5 according to the radiative constants $A_{2 a}$ and $A_{2 b} \cdot{ }^{27}$ This suggests that a strong self-absorption occurred in the plume. It is necessary to take account of the selfabsorption as well as the Stark effect for the analysis of the spectral line broadening observed in the present work. The best-fit results for the electron density parameter $N_{e 0}$, one of the three adjustable parameters, were less dispersed than the other parameters. In the calculation, the electron density is directly related to the Stark effect, which shows a line shift 


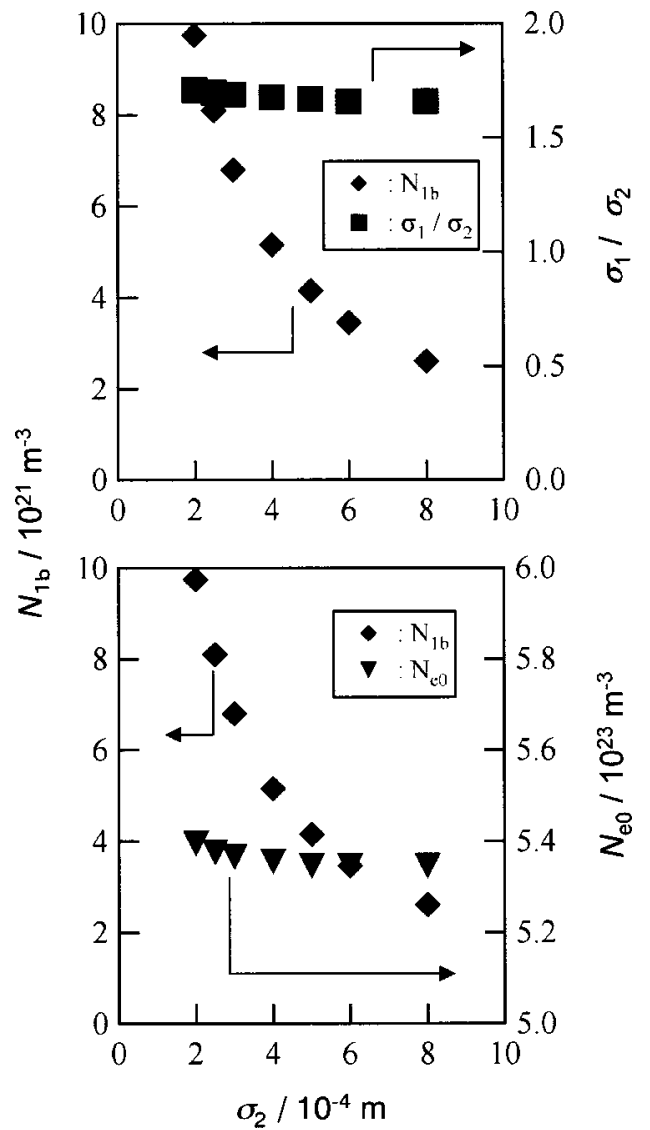

FIG. 7. The dependence of the best-fit parameters on the choice of $\sigma_{2}$ $\left(=\sigma_{e}\right)$. The examination was performed by using a spectrum obtained at $600 \mathrm{~ns}$ in 760 Torr of $\mathrm{N}_{2}$ gas.

as well as a line broadening. Particularly, the line shift is characteristic only of the Stark shift caused by the electron density, in contrast to the line broadening, which is influenced both by the self-absorption and the Stark effect. Therefore, there is no mixed contribution to the line shift, and the spectral-profile analysis with including the shift effect gives rather accurate value of the electron density. This leads to the less-scattered data shown in Fig. 3.

The reliability of the atomic density parameter $N_{1 b}$ and its spatial distribution parameter $\sigma_{1}$ is rather complicated. It has been shown that both $N_{1 b}$ and $\sigma_{1}$ are sensitive to the total line width and the depth of the self-reversed structure. ${ }^{26}$ This suggests that there are some correlations among the parameters $N_{1 b}, \sigma_{1}$, and $\sigma_{2}$. To verify this correlation, a model calculation was performed, where $\sigma_{2}\left(=\sigma_{e}\right)$ was varied within the range shown in Fig. 2, instead of fixing to an actually observed plume size. With assuming various values of $\sigma_{2}$, the parameters $N_{1 b}$ and $\sigma_{1}$ were obtained by fitting to a spectral profile observed at the delay time of $600 \mathrm{~ns}$ in $\mathrm{N}_{2}$ atmosphere. The results are shown in Fig. 7. For 2.0 $\times 10^{-4} \mathrm{~m}<\sigma_{2}<8.0 \times 10^{-4} \mathrm{~m}$, the ratio $\sigma_{1} / \sigma_{2}$ was constant at ca. 1.6. In addition, $N_{e 0}$ could be regarded as constant, i.e., the dependence of $N_{e 0}$ on $\sigma_{2}$ is negligible compared with the range of $N_{e 0}$ given in Fig. 3. On the other hand, $N_{1 b}$ decreased drastically from $1 \times 10^{22} \mathrm{~m}^{-3}$ to $3 \times 10^{21} \mathrm{~m}^{-3}$ with $\sigma_{2}$. It should be noted that the same calculations using a spectrum at another delay time give similar results.
The examination of the parameters suggests that the ratio $\sigma_{1} / \sigma_{2}$ determined by the analysis is rather reliable. The comparison between the observed and theoretical profiles gives good values of $N_{e 0}$ and $\sigma_{1} / \sigma_{2}$ regardless of the size of emission region $\sigma_{2}\left(=\sigma_{e}\right)$, while $N_{1 b}$ and absolute value of $\sigma_{1}$ are sensitive to $\sigma_{2}\left(=\sigma_{e}\right)$. The reliable determination of the atomic density parameter $N_{1 b}$ requires the correct value of $\sigma_{2}\left(=\sigma_{e}\right)$. In the present work, the size of the emission region was measured from the emission image at each delay time, and $\sigma_{2}$ determined from its size was used for the calculation. This procedure should give meaningful values of the parameters $N_{1 b}$ and $\sigma_{1}$, as well as $N_{e 0}$.

The dispersion of $N_{e 0}$ at each delay time was very small, while that of $N_{1 b}$ and $\sigma_{1}$ was comparatively large. This is probably due to the sensitivity of $N_{1 b}$ and $\sigma_{1}$ to $\sigma_{2}\left(=\sigma_{e}\right)$. The pulse-to-pulse fluctuation and the change of the target surface prevent us from an accurate determination of the size of the emission region, namely, $\sigma_{2}$. In the present experimental setup the plume imaging experiment cannot be carried out simultaneously with the measurement of the emission spectra, and hence, an average value of $\sigma_{2}$ at each delay time was used for the calculation. However, the size of the emission region for the particular ablation event at which the spectrum was obtained can be deviated from the average value of $\sigma_{2}$ used in the calculation. The comparatively large dispersion of $N_{1 b}$ and $\sigma_{1}$ is considered to be due to the error in the determination of $\sigma_{2}$.

\section{B. Ambient gas effect on the electron density}

It is clarified that the electron density parameter $N_{e 0}$ depends on the type of ambient gas species and its pressure. The effect of the pressure on $N_{e 0}$ (shown in Fig. 3) can be explained by the confinement of the plasma plume by an ambient gas. Here, we calculated the total number of free electrons in the plume, $N$, by assuming the Gaussian distribution of the electron density in the plume with the hemispherical symmetry,

$$
N=\int_{0}^{\infty} \int_{-\infty}^{+\infty} \int_{-\infty}^{+\infty} N_{e 0} \exp \left(-\frac{x^{2}+y^{2}+z^{2}}{\sigma_{e}^{2}}\right) d x d y d z .
$$

The total number of electrons in $\mathrm{N}_{2}$ atmosphere is shown in Fig. 8. The results for 760 Torr and 1520 Torr agreed within the uncertainty of the data. This result suggests that high $N_{e 0}$ values for 1520 Torr than for 760 Torr, as shown in Fig. 3, are explained by a strong confinement for 1520 Torr, which results in large $N_{e 0}$ but small $\sigma_{e}$ to give the total number of electrons to be the same as that of 760 Torr. On the other hand, we should point out another mechanism affecting the electron density, i.e., collision ionization could also affect $N_{e 0}$. The electrons emitted from the target collide with ambient species and then excite or ionize them. As a result, the number of electrons in the plume can depend on the atmosphere. Generally, the increase of ambient gas pressure, or the density of ambient species, causes the increase of collision frequency, and hence, enhances the ionization. According to the calculation of the total number of electrons shown 


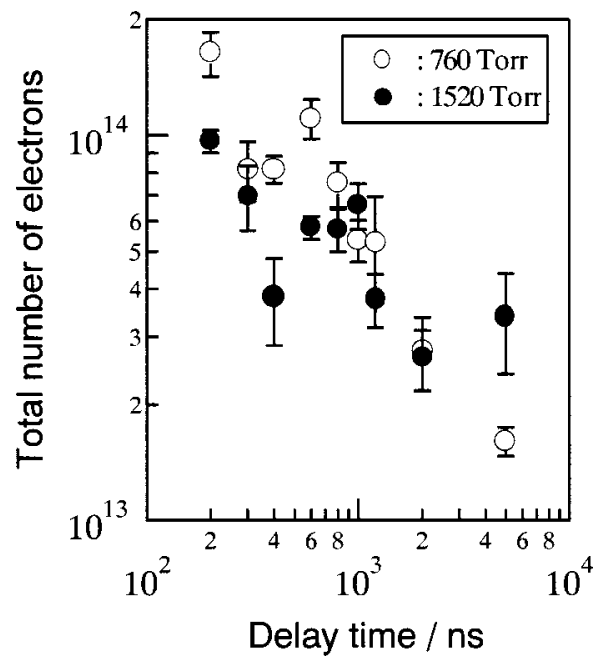

FIG. 8. Total number of electrons in the plume at 760 and 1520 Torr of $\mathrm{N}_{2}$ gas.

above, however, the results for the pressure effect can be explained mainly by the confinement effect, and the collision ionization seems to be a minor mechanism.

On the other hand, $N_{e 0}$ depends on the type of ambient species. As shown in Fig. 3, the larger size of ambient species brought about higher electron density in the order $\mathrm{He}$ $<\mathrm{CH}_{4}<\mathrm{N}_{2}<\mathrm{CF}_{4}$. As mentioned above, the plume confinement by the ambient gas can affect $N_{e 0}$. In He gas, the plume expanded more largely and rapidly than in other ambient gases. This can be the reason why $N_{e 0}$ in $\mathrm{He}$ was the smallest. However, although the plume size was similar for $\mathrm{N}_{2}, \mathrm{CF}_{4}$, and $\mathrm{CH}_{4}$ gases, $N_{e 0}$ in these gases shows the order, $\mathrm{CH}_{4}<\mathrm{N}_{2}<\mathrm{CF}_{4}$. This means that the ambient gas effect on $N_{e 0}$ cannot be explained only by the plume confinement. It is possible that $N_{e 0}$ is enhanced by the ionization of ambient species. Ionization cross sections of ambient species can be an indicator of the rate of this process. Although the cross section is a function of incoming electron energy and has a maximum value at around $100 \mathrm{eV}^{28}$ it increases with increasing molecular size, $\mathrm{He}<\mathrm{N}_{2}<\mathrm{CH}_{4}<\mathrm{CF}_{4}$, at any incoming electron energy, while the results of the present work show the $N_{e 0}$ order of $\mathrm{He}<\mathrm{CH}_{4}<\mathrm{N}_{2}<\mathrm{CF}_{4}$. We do not have an appropriate explanation on this discrepancy so far.

Regardless of the type of ambient gas, $N_{e 0}$ decreased rapidly with the delay time, and then leveled off. The rapid expansion suggests the adiabatic cooling. ${ }^{29}$ The cooling enhances the recombination in comparison with the ionization, and results in the decrease in $N_{e 0}$.

\section{The effects of ambient gases and their pressure on $N_{1 b}$ and $\sigma_{1}$}

As shown in Figs. 5 and 6, the time dependence of $N_{1 b}$ and $\sigma_{1}$ was not as clear as that of $N_{e 0}$, and their values have rather large dispersion. Slightly higher $N_{1 b}$ observed for the higher pressure is probably due to the confinement effect of the plasma plume. The dependence of $N_{1 b}$ and $\sigma_{1}$ upon the type of ambient species was not clear. This is consistent to the following explanation that the initial step of the ejection of surface species should not be influenced by the ambient

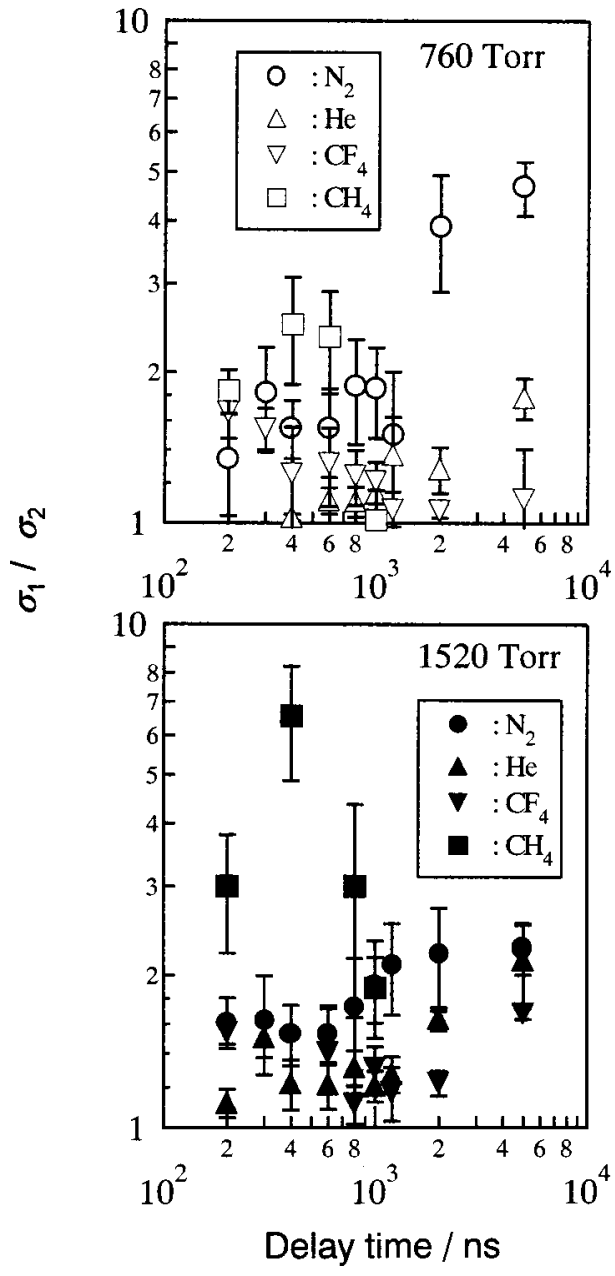

FIG. 9. The ratio $\sigma_{1} / \sigma_{2}$ obtained at 760 and 1520 Torr.

species. The time dependence of $N_{1 b}$ suggests that the formation of the ground state $\mathrm{Al}$ atoms continues until $1000 \mathrm{~ns}$. The relaxation of excited $\mathrm{Al}$ atoms, and/or electron-ion recombination of positively charged $\mathrm{Al}$ ions would contribute to the increase of $N_{1 b}$ till 1000 ns. However, a large distribution of the ground state $\mathrm{Al}$ atoms $\left(\sigma_{1}\right)$ in comparison with that of the plasma electrons $\left(\sigma_{e}\right)$ and the excited atoms $\left(\sigma_{2}\right)$ suggests another mechanism of the ground state $\mathrm{Al}$ formation. A possible explanation is that thermal evaporation of atoms lasts over $1000 \mathrm{~ns}$, and they stay in the plume. This explanation is also consistent with our recent work; the aluminum metal target gives larger distribution of the ground state $\mathrm{Al}$ atoms than the alumina target which has higher melting point and is hard to be evaporated thermally. ${ }^{30}$

Time evolution of $\sigma_{1}$ also did not show a big change by changing the type of ambient species or their pressure. Although the clear time dependence was not observed, it seems to show a slight increase except for $\mathrm{CH}_{4}$ gas. This suggests that the thermal evaporation continues within the time range in which the optical emission is detected. The ratio $\sigma_{1} / \sigma_{2}$ in each ambient gas shown in Fig. 9 did not strongly depend on the type of ambient species and their pressure, and was higher than unity. This assures that the temperature determined by the two levels involved in the transition is higher in the plume center than in the peripheral region. If the local 
thermodynamic equilibrium is achieved in this system, we can discuss thermodynamic behaviors of the plume by using the temperature distribution obtained by the present method.

\section{CONCLUSION}

Spectral line profiles of ablated species in laser-induced plasma plume were compared with theoretical spectra based on one-dimensional radiative transfer calculation, giving information about free electron density, atomic population density, and their spatial distributions. The electron density in the plume depends on the environment: the ambient species and pressure. Higher ambient pressure brings about a higher electron density due to the confinement effect. Also the electron density was affected by the ambient gas, i.e., He $<\mathrm{CH}_{4}<\mathrm{N}_{2}<\mathrm{CF}_{4}$. This was attributed to the ionization cross section as well as the confinement effect. In every ambient species, the electron density drastically decreased with time, and then leveled off. Atomic population density of the plume was also obtained from the spectral line profiles. Atomic population density was almost independent from the ambient species and pressure within the accuracy of the present data. It increased to a maximum value at around 1000 ns. The spatial distribution width of the atomic population of the lower level was ca. $1 \times 10^{-3} \mathrm{~m}$. It was always larger than that of the population of the upper level.

\section{ACKNOWLEDGMENT}

This work was financially supported by the Grant-in-Aid for Scientific research from Japan Society for the Promotion of Science.

${ }^{1}$ Q. Zhuang, K. Ishigoh, K. Tanaka, K. Kawano, and R. Nakata, Jpn. J. Appl. Phys., Part 2 34, L248 (1995).

${ }^{2}$ S. Amoruso, M. Armenante, V. Berardi, R. Bruzzese, G. Pika, and R. Velotta, Appl. Surf. Sci. 106, 507 (1996).

${ }^{3}$ Y. Franghiadakis, C. Fotakis, and P. Tzanetakis, Appl. Phys. A: Mater. Sci. Process. A68, 391 (1999).

${ }^{4}$ T. Venkatesan, X. D. W. A. Inam, and I. B. Wachtman, Appl. Phys. Lett. 52, 1193 (1988).
${ }^{5}$ V. Craciun and R. K. Singh, Appl. Phys. Lett. 76, 1932 (2000).

${ }^{6}$ A. Camposeo, F. Cervelli, F. Fuso, M. Allegrini, and E. Arimondo, Appl. Phys. Lett. 78, 2402 (2001).

${ }^{7}$ T. Makimura and K. Murakami, Appl. Surf. Sci. 96-98, 242 (1996).

${ }^{8}$ T. Sasaki, S. Terauchi, N. Koshizaki, and H. Umehara, Appl. Surf. Sci. 127-129, 398 (1998).

${ }^{9}$ K. Ogawa, T. Vogt, M. Ullmann, S. Johnson, and S. K. Friedlander, J. Appl. Phys. 87, 63 (2000).

${ }^{10}$ C. H. Bae, S. H. Nam, and S. M. Park, Appl. Surf. Sci. 197-198, 628 (2002).

${ }^{11}$ T. Sakka, S. Iwanaga, Y. H. Ogata, A. Matsunawa, and T. Takemoto, J. Chem. Phys. 112, 8645 (2000).

${ }^{12}$ T. Sakka, K. Saito, and Y. H. Ogata, Appl. Surf. Sci. 197-198, 246 (2002).

${ }^{13}$ K. Saito, K. Takatani, T. Sakka, and Y. H. Ogata, Appl. Surf. Sci. 197198, 56 (2002).

${ }^{14}$ T. Sakka, K. Takatani, Y. H. Ogata, and M. Mabuchi, J. Phys. D 35, 65 (2002).

${ }^{15}$ S. Iijima and T. Ichihashi, Nature (London) 364, 603 (1993).

${ }^{16}$ Y. Zhang, H. Gu, K. Suenaga, and S. Iijima, Chem. Phys. Lett. 279, 264 (1997).

${ }^{17}$ G. W. Yang and J. B. Wang, Appl. Phys. A: Mater. Sci. Process. A71, 343 (2000).

${ }^{18}$ G. W. Yang and J. B. Wang, Appl. Phys. A: Mater. Sci. Process. A72, 475 (2001).

${ }^{19}$ D. Lacroix and G. Jeandel, J. Appl. Phys. 81, 6599 (1997).

${ }^{20}$ S. S. Harilal, C. V. Bindhu, V. P. N. Nampoori, and C. P. G. Vallabhan, Appl. Phys. B: Lasers Opt. B66, 633 (1998).

${ }^{21}$ R. C. Issac, G. K. Varier, P. Gopinath, S. S. Harilal, V. P. N. Nampoori, and C. P. G. Vallabhan, Appl. Phys. A: Mater. Sci. Process. A67, 557 (1998).

${ }^{22}$ B. Toftmann, J. Schou, T. N. Hansen, and J. G. Lunney, Phys. Rev. Lett. 84, 3998 (2000).

${ }^{23}$ R. A. Lindley, R. M. Gilgenbach, C. H. Ching, J. S. Lash, and G. L. Doll, J. Appl. Phys. 76, 5457 (1994).

${ }^{24}$ N. Vogel and N. Kochan, Appl. Surf. Sci. 127-129, 928 (1998).

${ }^{25}$ J. Hermann, C. Boulmer-Leborgne, and D. Hong, J. Appl. Phys. 83, 691 (1998).

${ }^{26}$ T. Sakka, T. Nakajima, and Y. H. Ogata, J. Appl. Phys. 92, 2296 (2002).

${ }^{27}$ See Kurucz Atomic Line Database, Harvard-Smithsonian Center for Astrophysics, http://cfa-www.harvard.edu/amdata/ampdata/kurcz23/ sekur.html

${ }^{28}$ W. Hwang, Y. K. Kim, and M. E. Rudd, J. Chem. Phys. 104, 2956 (1996).

${ }^{29}$ S. S. Harilal, C. V. Bindhu, V. P. N. Nampoori, and C. P. G. Vallabhan, Appl. Phys. B: Lasers Opt. B66, 663 (1998).

${ }^{30}$ H. Furusawa, T. Sakka, and Y. H. Ogata, Appl. Phys. A (in press). 